\title{
Al encuentro del lugar: el caso de La parada del tonto en Concepción, Chile
}

Hugo Capellà. Universidad de Concepción, Concepción, Chile.

RESUMEN | Los lugares de encuentro se establecen en torno a nodos que terminan por convertirse en hitos colectivos, más allá de su forma. En el caso de La parada del tonto en la ciudad de Concepción, nos encontramos con un curioso ejemplo de un céntrico punto de encuentro que ha terminado por convertirse en un lugar con nombre propio.

En el presente artículo nos centraremos, en primer lugar, en la construcción de lugares desde su dimensión no solo espacial, sino también temporal, incidiendo en la importancia histórica que han tenido los relojes como hito temporal. En segundo lugar, nos adentraremos en el caso de estudio específico de La parada del tonto, de su transformación de nodo hasta convertirse en hito de referencia colectiva. Finalmente, concluiremos con una teorización sobre la dimensión universal del hito temporal, como sentido del lugar o topoi, cohesionador de un legado colectivo.

PALABras ClAVE | espacio público, imaginario urbano, patrimonio.

ABSTRACT | Meeting places are based on nodes that later become landmarks. La parada del tonto [The fool's stop] in Concepción is a peculiar example of how a central meeting point has become a place with its own name, with no relation to its surrounding urban form.

This article focuses firstly on how places are generated, according not only to the spatial dimension, but also to the temporal scale, associating place formation with the importance of clocks as significant aspects of spatial landmarks. Afterwards is an in-depth analysis of the case study, La parada del tonto, in order to understand how this space became a recognizable landmark. The article concludes with a theoretical development regarding the universal dimension of temporal landmarks as contributing to a sense of place or topoi, and providing cohesion as a collective legacy.

KEY WORDS | public space, urban imagery, heritage.

Recibido el 30 de julio de 2012, aprobado el 15 de abril de 2013

E-Mail: hcapella@udec.cl 


\section{El lugar de encuentro}

Difícil sería discernir si el lugar estimula el encuentro o bien si, por el contrario, son las sinergias colectivas las que terminan fraguando un lugar para el encuentro. En cualquier caso, existen sinergias colectivas que propician el auge de ciertos nodos como puntos de encuentro, aunque también estas dinámicas han sido estimuladas, o incluso establecidas ex novo desde la planificación urbana y, en especial, desde el diseńo urbanístico (Yates, 1966). Tras décadas volcadas por los planificadores al diseńo del espacio, se ha empezado a repensar los beneficios de los lugares en el diseño de las ciudades y, con ello, en la identidad urbana (Lalli, 1988). La consideración transversal y activa de las dialécticas de la ciudadanía con su entorno empieza a concebirse como el examen de un legado de experiencias que no podemos obviar a la hora de construir ciudad (Bettendorff, 2005).

La preocupación de urbanistas que, en los años sesenta, comenzaron a indagar en las relaciones entre las formas arquitectónicas y desde la perspectiva del contexto, permitió incorporar dimensiones sociales, históricas y psicológicas que no habían sido consideradas anteriormente. Se incluyó en este enfoque la observación de las realidades urbanas existentes, con la intención de categorizarlas y transformarlas en pautas para el diseńo urbano. Todo lo anterior con el objetivo de entender el diseño urbano como una función humana dinámica, creativa, estética, que refleja una verdadera participación ciudadana. El diseño urbano se constituye como una forma de crear espacio público, acorde con los places of the soul (Jacobs, 1958).

En cualquier caso, el lugar de encuentro en una ciudad responde a una necesidad social, y forma parte del concepto mismo de ciudadanía. Mantiene una estrecha relación con las formas de urbanidad, tradicionalmente asociadas al espacio público, en elementos como plazas o vías. Su existencia permite, en cierto modo, evidenciar esas formas de ciudadanía, y a la vez representa un legado esencial para su trasmisión como una forma de cultura urbana colectiva (Halbwachs, 1968).

En las ciudades, los lugares de encuentro han tendido a establecerse, desde la Antigüedad, en los espacios de mayor concurrencia y céntricos, en contra incluso de los propósitos de fluidez y funcionalidad que acarrea el aglutinamiento de personas en un mismo punto. Desde un punto de vista conceptual, la encrucijada simboliza el punto de convergencia y, por ende, el más propenso a convertirse en nodo y punto de encuentro. No obstante, en algunos casos a lo largo de la historia se han buscado lugares marginales para tales fines, como cerros o catacumbas, cuando la reunión involucraba a un grupo en particular que, de alguna forma, tenía el carácter de proscrito.

\section{La construcción de lugares}

Los lugares en la ciudad responden -como muy bien definió Lynch (1970) - a una lógica funcional y colectiva. Por lo tanto, para que un espacio urbano pueda ser considerado como lugar no importa solo su dimensión funcional, sino su representación, asumida como referente para el resto de la comunidad. Los hitos obedecen así no solo a una dimensión exclusivamente espacial, sino que se complementan con la coordenada de la dimensión temporal, representada por la propia comunidad. 
Las instancias del poder (político o religioso, o incluso civil) tuvieron una particular preocupación, desde los inicios de la urbanidad, por las escenografías o diseños para la dramatización simbólica de su señorío frente a los habitantes de las ciudades. El uso de los espacios públicos fue paralelo a la representación de las formas de poder. Este hecho conduce de manera normal a pensar que los lugares de encuentro se asocian de modo directo con los espacios de poder. No obstante, esta correlación no es generalizable, ya que si bien es cierto que la mayoría de los espacios de poder se han concebido como lugares de reunión, no podemos aseverar que todos los lugares de encuentro tengan que vincularse con el poder. En muchas ocasiones, el temor al poder ha llevado a la búsqueda de lugares de encuentro tolerados, aunque al margen. En definitiva, si bien podemos incentivar o motivar espacios como puntos de encuentro, difícilmente podremos fijar de antemano tales lugares en una ciudad, porque obedecen a las decisiones de la colectividad. Esto nos permite entender cómo ciertos espacios periféricos pueden terminar siendo lugares de encuentro y, por el contrario, cómo ciertos espacios centrales, como una plaza, pueden ser solo espacios simbólicos de la representatividad oficial y del poder. No obstante, ciertas instancias oficiales muchas veces han adecuado las formas de lugares de encuentro bajo el alero de una tolerancia implícita.

Para existir como tal, el lugar de encuentro debe conjugar la dimensión espacial, al plantearse como nodo funcional, así como una dimensión temporal acorde al referente colectivo. Esta segunda dimensión, la temporal, configura la memoria colectiva que define la particularidad del lugar. De hecho, la mayor crítica que se ha hecho a los diseñadores urbanos remite a tal aspecto, en el sentido de que confunden el place of sense generalizador con el sense of place, particular, reflejo del referente colectivo (Jacobs, 2004).

La correlación del referente temporal con la dimensión espacial urbana ha constituido una preocupación desde las primeras formas de urbanización, como manera de compensar y aglutinar a las comunidades. La representación del tiempo universal y cronológico se ha reflejado en hitos urbanos que condicionan muchos de los lugares de encuentro. Así, la ubicación de relojes inserta una dimensión temporal en el espacio, que aglutina a las colectividades en sus encuentros. El papel de la dimensión temporal en la articulación de lugares por la sociedad ha sido planteado como heterocronía (Nogué, 2007).

La construcción de los lugares desde la participación de sus habitantes, y desde una visión dinámica, permite establecer (re)significaciones múltiples y complejas, incluyendo heterotopías (Nogué, 2007). El lugar se constituye como un referente colectivo que adquiere relevancia patrimonial. La transmisión oral colectiva de los testimonios referidos a tales patrimonios representa un legado que forma parte de la identificación con la ciudad en que ellos existen (Francis, 1983). Los lugares, entendidos desde su dimensión física pero igualmente como representaciones icónicas colectivas (Valera, 1996), participan en la construcción de la memoria colectiva, como define Nora en su texto Los lugares de memoria (2009). En el presente caso de análisis de La parada del tonto, el referente no solo se puede entender como un lugar para la memoria colectiva penquista, sino que metafóricamente podemos comprender que, tras el topónimo vinculado al lugar, existiría una forma de 
memoria del lugar. La pervivencia y transmisión del nombre de los lugares (topónimos) significa no solo la pervivencia de un referente colectivo urbano, sino que también constituye, en el caso de Concepción, una forma de patrimonio invisible y flexible perdurable por su transmisión colectiva, que se adecua al carácter efímero de las formas físicas, sujetas a los caprichos de una considerable naturaleza telúrica como la propia de la región. La memoria de los lugares representa la identidad como referencia colectiva en un compromiso necesario para las sucesivas reconstrucciones materiales. La herencia del referente transmitido (la memoria) es la que permite la permanencia física del lugar.

El presente caso de estudio permite recalcar justamente la discusión planteada desde la psicología (Aguilar, 1990) en torno al grado de importancia del ambiente no solo en la formulación de los referentes individuales, sino también en la conducta colectiva y urbana (Corraliza, 1987). El caso penquista ilustra justamente cómo esas representaciones sociales no se apoyan en general sobre realidades físicas -el builtscape (Aragonés, 1992)-, sino que incluso pueden convertirse en la causa de su permanencia física. La relación entre representación y realidad se invierte. La representación legada por el grupo se convierte en este caso en la razón para la reconstitución de la realidad urbana construida. El empeńo de pervivencia, grado de responsabilidad colectiva y compromiso adquieren un tono casi épico frente a los distintos episodios traumáticos -terremotos y maremotos- que ha vivido la ciudad, situaciones de las que cada habitante se siente parte y referente individual, y también en cuanto colectivo. El proceso de reconstrucción desde el lugar de la memoria se convierte a la vez en un acto de autoafirmación colectiva del gentilicio, que se ve renovado.

\section{El reloj, un referente universal}

El control matemático del tiempo universal se convirtió en una forma de control sobre las pautas de vida $\mathrm{y}$, finalmente, en una forma de imposición precisa. La complejidad del manejo astral y temporal quedó bajo el alero del poder (religioso y político) como una forma de dominio sobre sus habitantes. Desde hace poco menos de cien ańos el conocimiento de la hora se ha estandarizado con los relojes de pulsera e implementos tecnológicos contemporáneos, como celulares. Todo ello nos hace olvidar la importancia del manejo y dominio del tiempo. En el mundo contemporáneo hemos conseguido democratizar el acceso a la información de la hora, aunque la creciente precisión se ha transformado en una nueva forma de control sobre nuestras vidas. El mayor grado de detalle y universalidad de la hora la convierten en una obligación que pauta las formas de relacionarnos.

Lejos de lo que se podría imaginar, el control del tiempo desde el poder en la ciudad se remonta a la Antigüedad, con las primeras clepsidras (reloj de agua: ydor, agua, y clettó, ocultar), como la del ágora (siglo IV a. C.) o la clepsidra del ágora romana (Foro) en Atenas, conocida como Horologion de Androniki (50 a. C.) o Torre de los Vientos (Figura 1).

La preocupación por el tiempo en el espacio se vio reflejada en la instalación de clepsidras. Estas se situaron siempre en un ángulo de las ágoras, por un lado marcando simbólicamente la coordenada espacio-temporal necesaria para fomentar 
los lugares de encuentro colectivo; y por otro, incentivando alguna de las primeras formas de diseño urbano como estrategia de control sobre la ciudadanía. La Torre de los Vientos no solo servía como reloj, sino como una suerte de estación meteorológica, con indicaciones sobre los vientos, y ante los ojos de los habitantes de Atenas representaba una forma de dominio de las vanguardias tecnológicas de la época, fomentadas desde el poder, sobre la ciudadanía.

Más allá de su dimensión política y simbólica, interesa recalcar la estrecha relación entre el reloj y la plaza y, en particular, entre un ángulo de la plaza y el hito del reloj. A pesar de poder parecer casi accidental, esta característica se mantendrá a lo largo de la historia hasta la actualidad, incluso en nuestro caso de estudio en Concepción.

\section{Figura 1 | Clepsidra en el foro romano de Atenas (siglo I a. C.)}

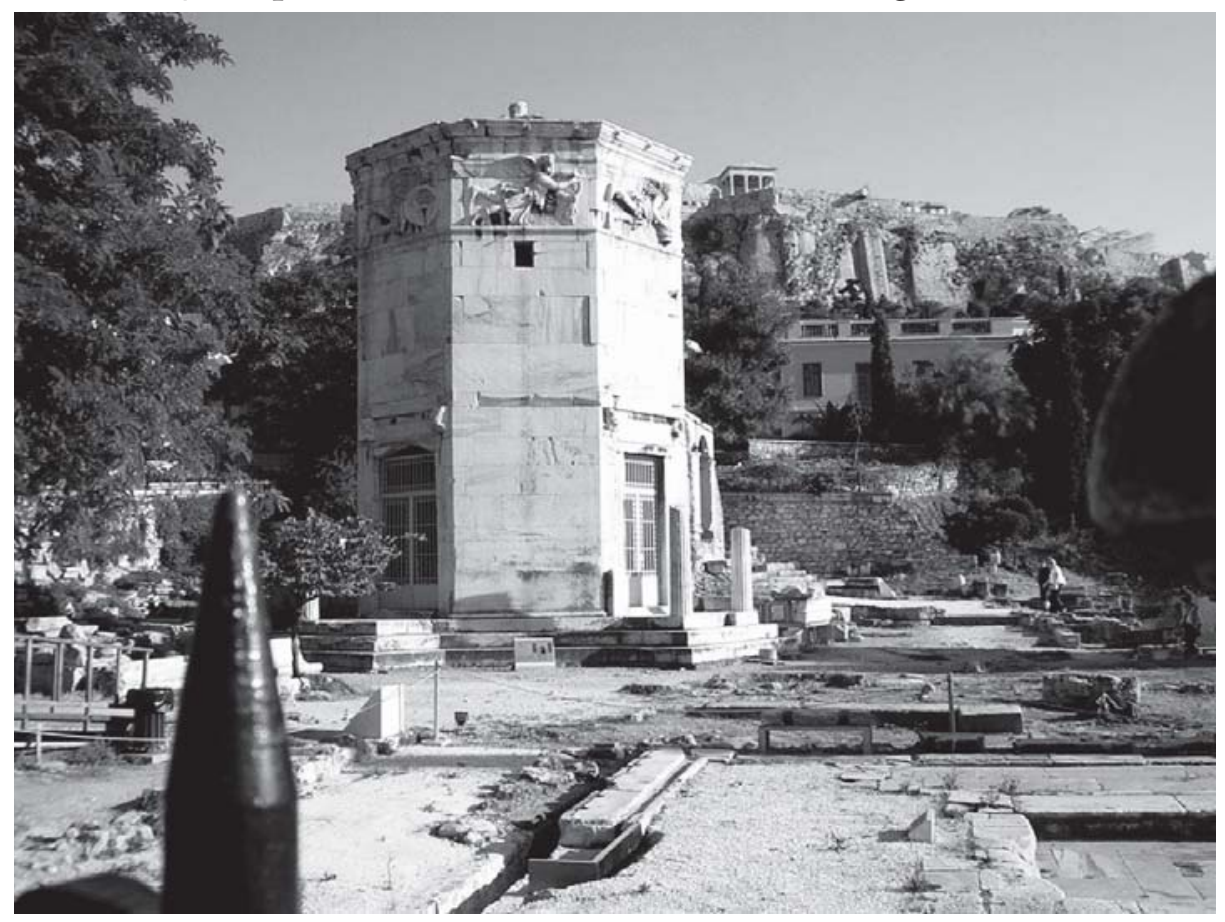

FUENTE FOTOGRAFía DE JAVIER MARTÍN-ARTAJO GUTIÉRREZ (AAS). EL REFERENTE TEMPORAL EN EL LUGAR TIENE UNA LARGA TRADICIÓN

La esquina de la plaza y el reloj representan la unión óptima de las dimensiones espacio-temporales para establecerlo como lugar de encuentro ciudadano (Lynch, 1972). El carácter lateral de la esquina y casi marginal respecto de la plaza central, encierra en el fondo una simbolización voluntaria en la construcción de un hito de tolerancia para la ciudadanía. El carácter lateral evita la obstaculización y fomenta la reunión ciudadana. La ubicación de los horologion fue concebida desde un diseño intencionado, pero con el objeto de parecer casual, para que pudiera ser apropiado por la ciudadanía. El lugar en la urbe aparece como una construcción fruto del azar, lo que constituye parte de su éxito. 


\section{La tradición urbana de los relojes}

El uso de los relojes públicos en Occidente se propagó a partir del siglo XIV, con los horologium (del griego del de Padua en Italia. Los primeros relojes se van a vincular con edificios oficiales. La hora venía dictada desde las iglesias, que ya pautaban el tiempo y las alertas con sus campanas, así como de instancias del poder, como palacios reales: es el caso del Louvre, por ejemplo. El control del reloj y de la hora por parte del monarca, en ese caso, implicaba una simbolización del poder que se proyectará, en el caso de París, sobre un eje de perspectiva hacia el Oeste para el crecimiento de la ciudad (Campos Elíseos). El palacio del Louvre representaba la coordenada espacio-temporal que define el hito del poder, el Este.

La aparición de relojes en edificios civiles, como ayuntamientos en Flandes, simbolizará una forma de secularización del tiempo a manos de una ciudadanía libre. De hecho, en algunos lugares el reloj ha fijado más recientemente no solo el lugar de encuentro diario, sino que se ha tomado como escenario simbólico de la celebración de los ciclos anuales. En el caso de la Plaza del Sol de Madrid, por ejemplo, la plaza se convierte en el lugar simbólico desde la dimensión espacial (al ser el kilómetro cero de la red de carreteras nacionales), así como en su dimensión temporal, al marcar el tiempo oficial y el ciclo anual con la celebración del cambio de ańo.

En los tiempos modernos, los relojes se irán difundiendo en las ciudades, democratizando la hora. Las estaciones de ferrocarriles, como nuevos templos de la modernidad, reflejarán en sus enormes relojes la imposición de nuevos modos de vida, regidos por la precisión y optimización de los tiempos. Los relojes se convierten en instrumentos funcionales que reglamentan las salidas y llegadas de trenes, mientras en la puntualidad de los servicios versa la seriedad y honor de los nuevos poderes nacionales. Pensemos en la emblemática puntualidad británica o germánica, convertida en virtud nacional.

El reloj se convierte en el elemento omnipresente en el mobiliario urbano y se vincula estrechamente con el punto de encuentro.

El caso del café Zúrich es un buen ejemplo. La esquina de este renombrado café barcelonés, situado en una esquina de la plaza de Catalunya y nodo clave con la continuación de las Ramblas, se ha convertido en el lugar de encuentro por excelencia de sus habitantes, aunque hoy pocos recuerden el reloj situado encima del establecimiento, testimonio mudo de la antigua estación de tren ubicada en esa esquina y que luego sería soterrada. El lugar de encuentro quedó fraguado en esa esquina, como conjunción espacial y temporal, y se ha convertido en parte de un legado, como hito colectivo, asociado en este caso a un café.

\section{Plazas y relojes}

La relación de las plazas y los relojes, como ya se ha visto, se remonta a la Antigüedad y es mucho más estrecha de lo que pudiera parecer en primera instancia. Representa la conjunción del espacio y del tiempo para configurarse como referente e hito colectivo. La relación, si bien parece evidente, no aparece urbanísticamente de forma explícita, ya que la centralidad espacial opaca muchas veces el carácter periférico de la dimensión temporal. El tiempo en la morfología urbana queda anclado 
en una de las esquinas de la plaza, dando un carácter periférico y libertario al punto de encuentro. Los lugares de encuentro aparecen así como accidentales y casuales. Difícilmente podríamos reconocer que son, en realidad, un elemento común a casi cualquier ciudad, no el fruto de las particularidades ciudadanas. Así, por ejemplo, la esquina de Times Square simboliza en su nombre la idea del lugar, al aludir al espacio Square (plaza) y al tiempo Times. La plaza del tiempo que se reduce además urbanísticamente a una esquina, y se vincula a un reloj que es igualmente tomado como escenario simbólico del cambio de ciclo anual por sus ciudadanos.

La relación del tiempo con el espacio encierra en el fondo una cohesión intrínseca del lugar. El carácter casual del encuentro entre ambas dimensiones garantiza el éxito del referente colectivo. El punto de unión se manifiesta siempre en un reloj ubicado en el ángulo de una plaza y junto a la arteria con mayor flujo humano. En esta situación queda la duda de si se trata de una casualidad o bien de una intencionalidad en el diseño y control del hito del lugar. En este contexto podemos entonces hablar del lugar como un hito colectivo o bien debemos entenderlo como un símbolo de referencia colectiva. El caso que explicaremos a continuación resulta de la creación ciudadana de un hito legado y que se reduce a un nombre dado.

\section{La parada del tonto: un referente urbano}

La parada del tonto o Parada de los tontos es el nombre dado a una esquina del centro de Concepción que se usa como punto de encuentro. El nombre refiere al hecho de la espera de pie como "tonto", mientras aparece la otra persona, ya que el "otro" nunca es puntual. La expresión coloquial, que podría parecer actual y reducida a un público joven, resulta encerrar una larga tradición y se vincula con la instalación del reloj en la antigua Municipalidad, situada en esa esquina. La frase, en su alusión a la espera en el encuentro, aparece también en expresiones de otras culturas, como en la arquitectura palaciega francesa, donde las salas de espera antes de una audiencia son denominadas salles des pas-perdus [salas de los pasos perdidos], en alusión a los pasos que se dan en vano mientras se espera. En ambos casos, las locuciones referidas al lugar remiten en cierto modo a una desorientación, aunque causada por la espera.

La parada del tonto ilustra el caso de la construcción de un referente urbano colectivo, establecido sobre un nombre dado y transmitido con independencia de la forma específica del lugar. Esta particularidad obedece, en parte, al contexto sísmico de la ciudad, que ha establecido una memoria colectiva construida sobre la trans-

misión de referencias a lugares independientes de sus formas efímeras, a diferencia de aquellos casos en que las referencias son a sitios estables en el tiempo (Mazzei de Grazia, 2004).

Retomando los conceptos acuñados por Lynch (1960), veremos cómo el punto de encuentro se establece espacialmente como un nodo, en el sentido de que es un punto focal, establecido como intersección. Puede definirse como lugar por su dimensión temporal, al estar vinculado con un reloj. Cuando nos referimos a $\mathrm{La}$ parada del tonto aludimos al nombre dado colectivamente a esa localización, que se identifica como referente urbano y se considera como hito para la comunidad. El hito adquiere una dimensión temporal por su transmisión intergeneracional, y 
en este caso se ha convertido en un referente que identifica la ciudad con sus habitantes, que les da una identidad como "penquistas". Solo se juntarán en La parada del tonto aquellos que conozcan el nombre y sepan ubicar dicho lugar, porque para el resto es imposible reconocerlo por el nombre. La expresión se convierte en un topónimo que forma parte de la identidad penquista.

\section{El punto de encuentro: un nodo}

El punto de encuentro de La parada del tonto es una intersección entre las calles Barros Arana y Aníbal Pinto en el centro de Concepción (Figura 2). Se sitúa en uno de los vértices de la plaza de la Independencia (plaza de Armas central del modelo en damero tradicional colonial, construida tras el traslado de la ciudad en 1752 desde su antiguo asentamiento en la actual ciudad de Penco, luego del terremoto y maremoto de 1751) y sobre dos de las principales arterias comerciales, en la actualidad parcialmente peatonalizadas (Alarcón, 1998).

FIgURA 2 | La parada del tonto: de nodo a hito

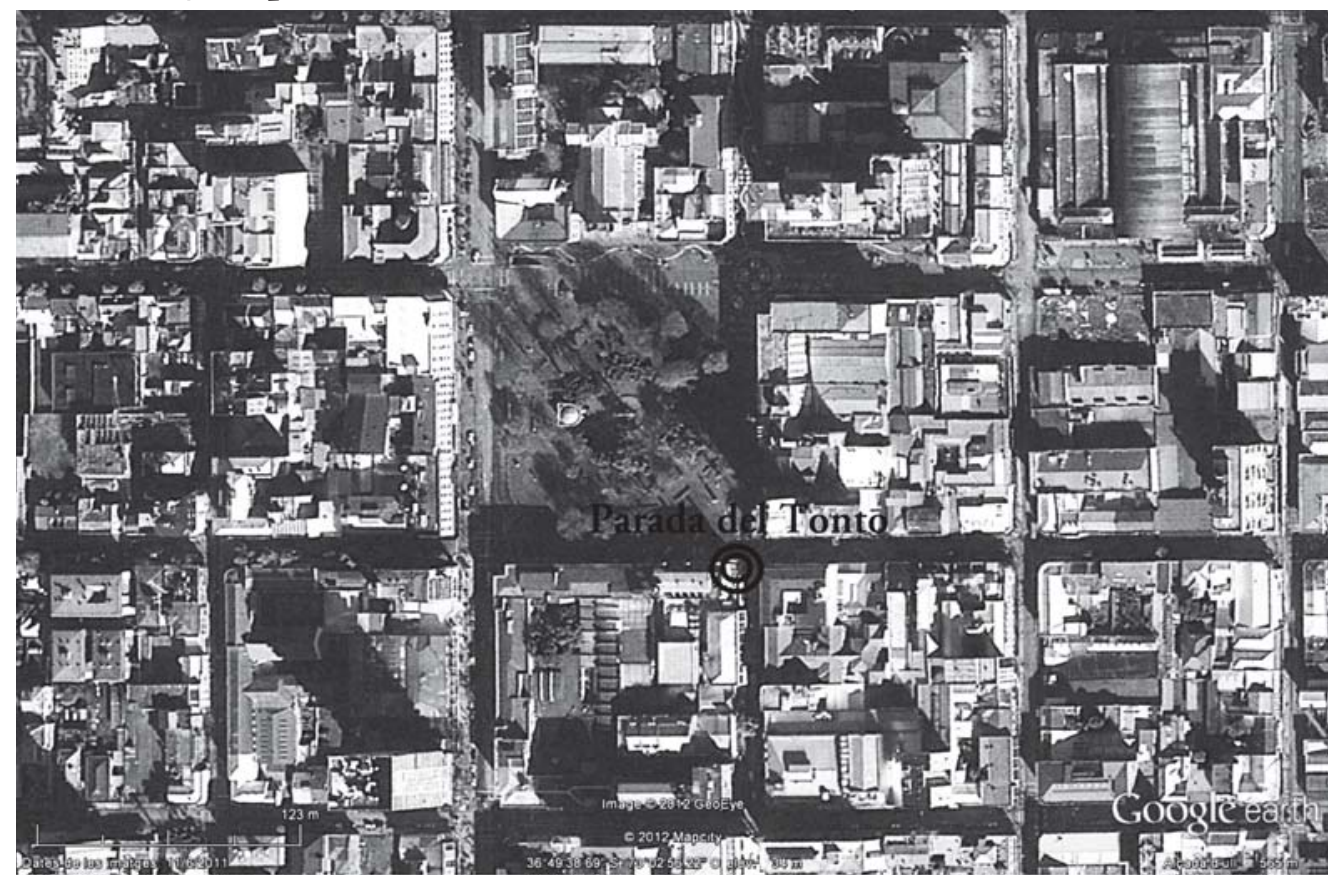

FUENTE GOOGLE EARTH

La permanencia del lugar de encuentro en Concepción remonta a principios del siglo xx, con la instalación del edificio de la antigua Municipalidad (Figura 3) en la denominada La esquina de la puñalá y, más recientemente, La parada del tonto, desde la peatonalización de la calle Barros Arana con el paseo Alonso Ercilla Zúñiga (1981-1990). La mejora del diseńo urbano de la plaza en 2004-2005, con la instalación del reloj, así como la aparición de una gran pantalla gigante Led (2007) (Oliver \& Zapata, 1950) han consolidado el hito. 
La historia de la ubicación de la Municipalidad de Concepción refleja, en buena medida, la trágica historia urbanística de la ciudad, siempre sujeta a terremotos de gran magnitud, con un promedio de dos megaterremotos por siglo. Desde su fundación colonial (siglo xvI) los testimonios escritos recogen las consecuencias destructoras de cada sismo, así como los complejos procesos de reconstrucción de la ciudad, que -como está dicho- incluso llevó en 1752 a su relocalización alejada de la costa, para evitar al menos los embates de los maremotos (Lara, 1998). El edificio de la Municipalidad, tras distintas ubicaciones, fue reconstruido en 1916 en el cruce de las actuales calles Barros Arana y Aníbal Pinto, justo frente a la plaza de la Independencia y al lado de la Intendencia. El edificio, de influencias arquitectónicas francesas, estaba coronado por un reloj (Pacheco, 1997).

\section{Figura 3 | Esquina de la antigua Municipalidad de Concepción (1916-1968)}

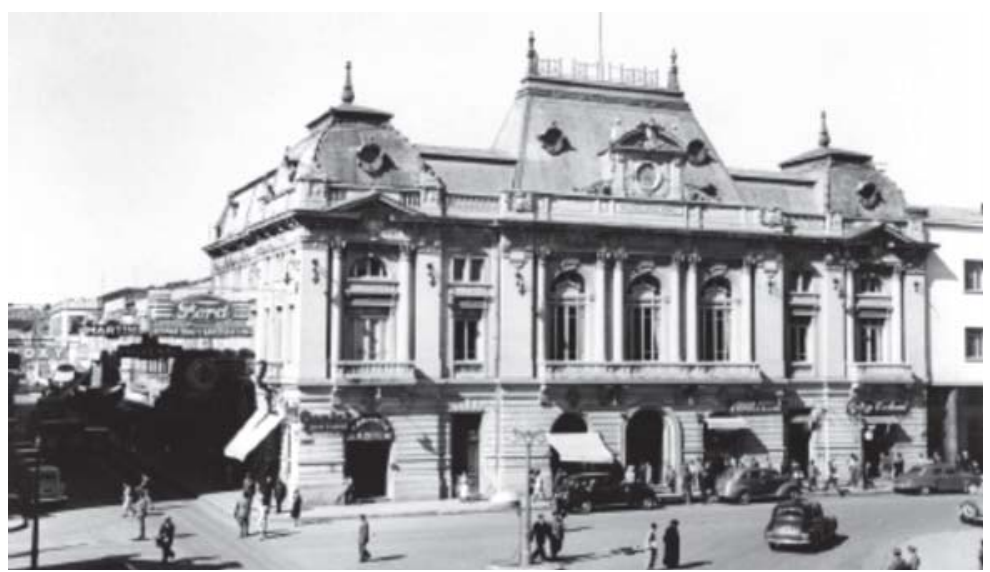

FUENTE ARCHIVO FOTOGRÁFICO ALEJANDRO MIHOVILOVICH. ESTA ESQUINA SE CONVIRTIÓ EN EL PUNTO DE ENCUENTRO E HITO URBANO CONOCIDO COMO LA ESQUINA DE LA PUNALLÁ Y ULTERIORMENTE COMO LA PARADA DEL TONTO

La belleza del edificio, el reloj, así como su ubicación en una esquina de la plaza y en una calle principal, llamada hasta 1906 calle del Comercio (hoy Barros Arana), lo convertían en un lugar céntrico, de fácil acceso y detección para encontrarse (Campos, 1985).

Este último piso es bastante cómodo, de buena altura y con grandes lucernas en los torreones del techo, que lo llenan de luz y mantienen una constante ventilación. En el centro se colocará un gran reloj de metro y quince centímetros de diámetro y que se moverá por medio de un pequeño motor eléctrico. Su instalación demorará algún tiempo, por haber sido encargado recientemente al extranjero... (Rolleri, 2007).

Lamentablemente, poco después de su inauguración en 1916, el edificio padeció desperfectos con el terremoto de 1939 que obligarían al traslado de la Municipalidad, y posteriormente, tras el terremoto de 1960, terminarían por obligar a su demolición en 1968. Ello ocurrió a pesar de la negativa popular ciudadana, muy apegada a ese edificio, e incluso tras alguna consulta popular organizada por la prensa local de la época. El lugar de encuentro persistió más allá de la desaparición de la 
Municipalidad, por la transmisión de la tradición local de seguir encontrándose en ese mismo punto (Figura 4).

Por Aníbal Pinto estaba la Municipalidad, elegante construcción de estilo francés de fines del siglo pasado; la Corte de Apelaciones y el imponente y hermoso edificio de la Intendencia, de severas líneas arquitectónicas clásicas, que ocupaban toda la cuadra. En los bajos de la Intendencia estaban las oficinas y en los altos la morada de la primera autoridad provincial... (Louvel, 1995).

Louvel, en sus memorias sobre la plaza de la Intendencia realizadas a principios del siglo xx, parte su descripción, curiosamente, de la esquina de Barros Arana con Aníbal Pinto, subrayando la relevancia simbólica y referencial de ese punto en particular por sobre el resto de elementos emblemáticos. En su crónica se confirma tal idea cuando al referirse al negocio del Lustrín de Merino:

En uno de los locales comerciales ubicados en los bajos del antiguo y tradicional edificio de la Municipalidad, de puro estilo francés de fines del siglo pasado, estaba el famoso depósito de diarios, revistas y libros del recordado hombre de negocios y probo vecino de nuestra ciudad, don Rafael Merino, propietario también de una librería situada en O’Higgins, entre Pinto y Colo Colo, al lado del solar que ocupó, hasta hace unos años, la Sociedad de Empleados de Comercio.

En el depósito mencionado existió, además de los diarios y revistas, el famoso "lustrín" llamado festivamente "la esquina de la puñalá, porque allí se reunían jóvenes y hombres maduros en busca de la atención del lustrado de sus botines y, entretanto, se ocupaban en comentar las pequeñas cosas del diario vivir citadino. La sección del local dedicada al lustrín se componía de sillones, no exentos de cierta comodidad, bajo los cuales había dos dispositivos de bronce fundido, a manera de zapatos, que servían para apoyar los pies, mientras los lustrabotas se dedicaban seriamente a su oficio.

Antes de atender a un cliente y una vez sentado este, le proporcionaban con la mejor de las sonrisas una ajada revista, con manchas indelebles de anilina de todos colores, la que se agradecía sin siquiera mirarla a veces, mientras los zapatos, con tierra del verano o barro en el invierno, eran sometidos al procedimiento del lustreo.

Allí había, al menos, ciertas comodidades; se estaba bajo techo y los lustrabotas se dedicaban a su trabajo. En tanto que hoy los muchachos que hacen esta misma labor en la plaza, mientras lustran miran de un lado a otro, se distraen, pasan pomada sobre los calcetines y, entre ellos, comentan sus aventuras, algún partido de fútbol o el baile en perspectiva; todo esto no siempre acompañado ciertamente de un idioma muy académico... (Louvel, 1995, versión electrónica). 
FIGURAs 4 y 5 | La esquina de La parada del tonto

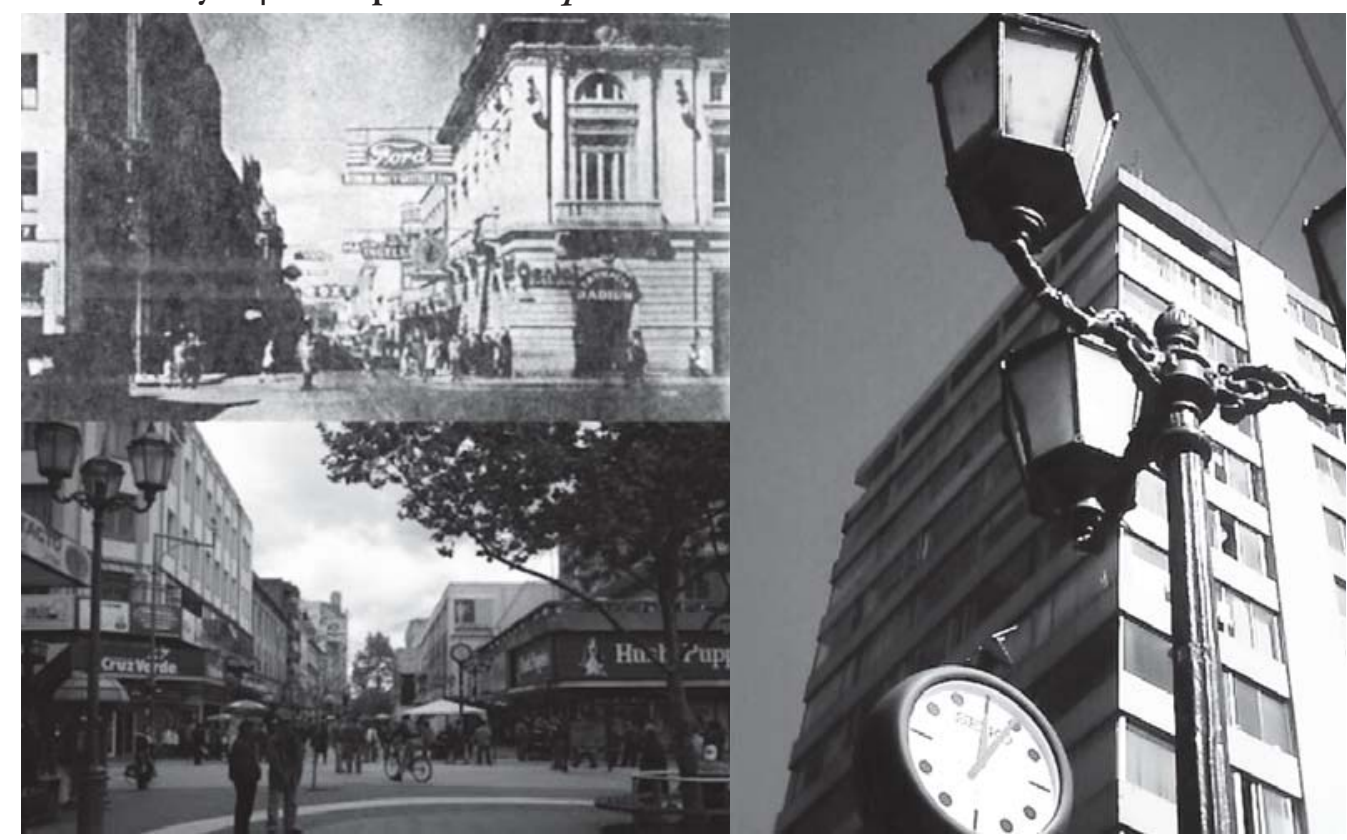

FUENTE FOTOGRAFÍA DE LUIS DARMENDRAIL Y GUILLERMO ROX. ESTA ESQUINA HA VARIADO A LO LARGO DEL TIEMPO, PERO EL PUNTO DE ENCUENTRO PERMANECE. IMAGEN DE LA IZQUIERDA: ES UN MONTAJE DE LA ESQUINA EN LOS AÑOS VEINTE Y EN LA ACTUALIDAD. IMAGEN DE LA DERECHA: ILUSTRA EL RELOJ QUE SE ENCUENTRA EN LA ACTUALIDAD EN LA ESQUINA DEL PUNTO DE ENCUENTRO Y QUE HA DADO ORIGEN AL NOMBRE ACTUAL DE LA PARADA DEL TONTO

La descripción del negocio de lustrador recalca el carácter emblemático de la esquina que servía de punto de encuentro para los varones. Mientras esperaban al frente de la Municipalidad, aprovechaban el tiempo para lustrar sus zapatos e intercambiar impresiones. No es de extrañar, entonces, el clamor popular ulterior y resistencia ante la demolición del edificio que ocupaba esa esquina, en 1968.

La peatonalización de la calle en los años noventa permitió ir incorporando, desde el diseńo urbano, elementos que reconocieran este referente colectivo en su nueva denominación, como La parada del tonto (Figura 5). La instalación de uno de los relojes de la plaza en dicha esquina, así como más recientemente la ubicación de una pantalla gigante de TV para la gente que espera, acentuaron el carácter de reunión de dicho lugar. Lamentablemente, el último terremoto, en 2010, debilitó uno de los pocos edificios aún existentes en esa esquina de principios del siglo $\mathrm{xx}$, el hotel Ritz, lo que obligó a retirar la pantalla (Figura 6).

El espacio de este punto de encuentro se ha visto modificado de forma continua por la historia trágica de la ciudad, aunque ello no ha incidido en la dinámica del lugar: el nodo ha persistido más allá del tiempo, gracias en gran parte a ser un hito colectivo. La consideración y transmisión del significado de ese lugar lo ha convertido en un referente de la identidad penquista. De hecho, el mismo gentilicio de los habitantes de Concepción alude al primer asentamiento de la ciudad en Penco y demuestra, en cierto modo, cómo el nombre y el referente persisten más allá de las circunstancias de su forma, gracias al esfuerzo y significado colectivo. En el caso de estudio, la pervivencia del referente colectivo en la conciencia de la comunidad 
local ha permitido la readecuación de la forma espacial de este punto de encuentro. La memoria colectiva, con sus olvidos incluidos, garantiza la continuidad de este espacio, como lugar (Ricoeur, 1999).

\section{FIGURA 6 | Imagen panorámica de la esquina de La parada del tonto}

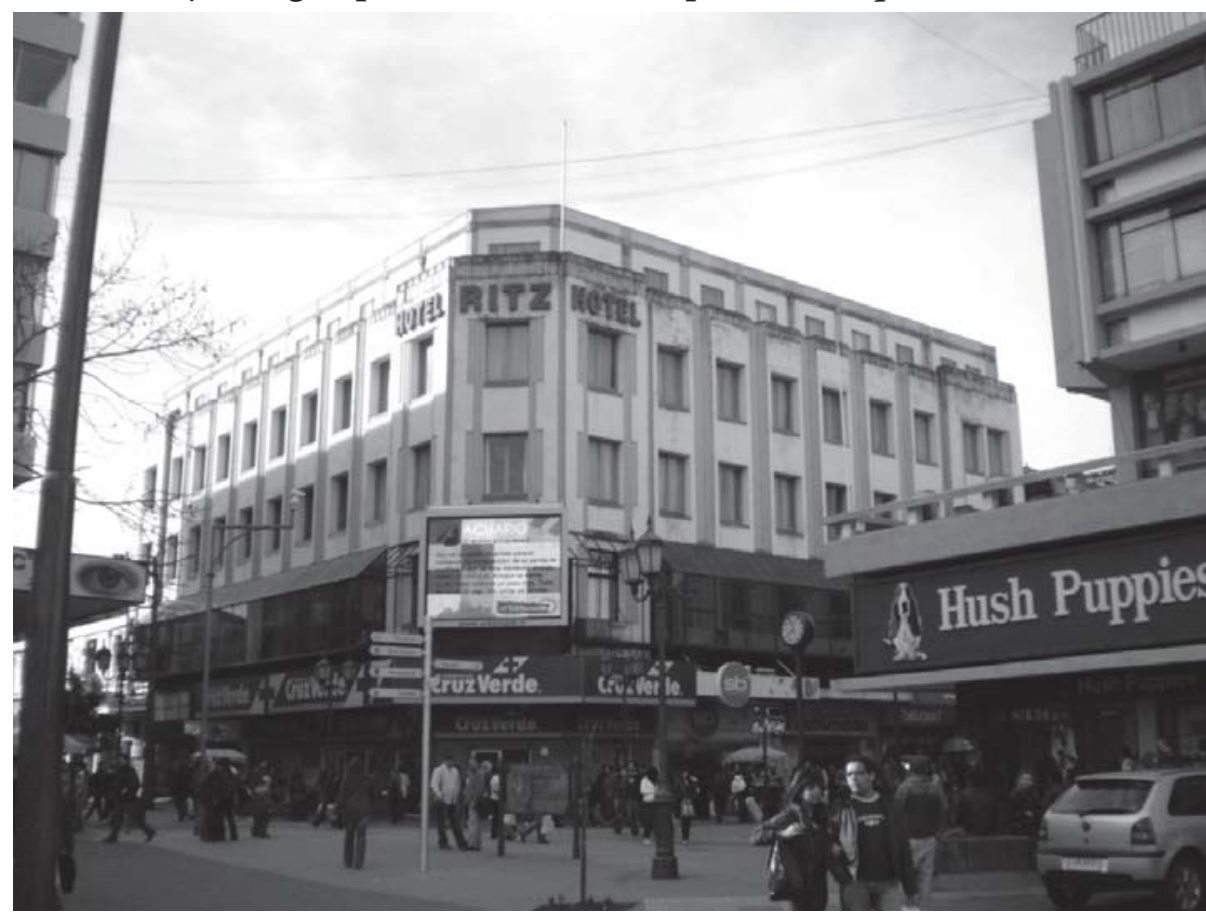

FUENTE FOTOGRAFÍA DE PUPA KRAMCSAK. EN LA IMAGEN PUEDE VERSE LA PANTALLA GIGANTE LED, QUE DESAPARECIÓ DESPUÉS DEL TERREMOTO DE 20 IO

\section{El referente urbano: un hito}

Las autoridades locales han estado considerando recientemente la puesta en valor del lugar en su diseño urbano, aunque ya existía de antes. La existencia de testimonios orales locales permite corroborar cómo esa esquina ya era considerada como punto de encuentro mucho antes de su implementación urbana actual, con la instalación del reloj. Su valorización urbanística debe ser, por lo tanto, entendida como una consecuencia que permite acentuar las interacciones que ocurren en el lugar.

La originalidad de La parada del tonto reside en el hecho de que su establecimiento como hito se fundamenta exclusivamente en la transmisión oral que la comunidad ha hecho de su función como punto de encuentro, sin que ello tenga vinculación con elemento espacial alguno. El nombre dado al lugar se convierte en el referente y pasa a considerarse como un hito por sí mismo. El carácter patrimonial de ese hito reside en la dimensión de legado, transmitido en el tiempo de forma colectiva.

¡La parada de los tontos! Esto es Aníbal Pinto con Barros Arana, justo donde están los árboles es la plaza de la Independencia (ahí se dicto la independencia de chile es la única plaza en chile que tiene diferente nombre, las demás plazas son plaza de armas), no esta así de lleno siempre pero es que es el paradero de los estudiantes y le dicen la parada de los tonto, porque como buen chileno nadie es puntual así 
que la otra persona se queda esperando, así como tonto... (Blogger http://mixcool. blogspot.com/2005/08/concepcin-lugar-para-vivir.html)

La Pará del Tonto: Lugar de la ciudad (Barros esquina Anibal Pinto) en donde el tiempo se detiene y te quedas pegado mirando al reloj existente que tiene la misma hora a cada momento, porque no funciona. O sea, jelou, eres un tonto por quedarte mirando un reloj que no sirve de nada. En diciembre del 2007 se instalo en este lugar un tremendo televisor (al estilo del Paseo Ahumada de Santiago), donde pasan puros comerciales; por lo que ahora la gente se queda mirando esa tele (y parecen mas wnes todavía k mirando el reloj)... (http://www.pediars.com/wiki/Concepci\%C3\%B3n)

Estos son algunos de los testimonios que aparecen en redes sociales y reflejan el carácter referencial de ese lugar ${ }^{1}$. La mayoría de los penquistas termina por incluir ese hito en las páginas oficiales como uno de sus atractivos turísticos (Figura 7).

El conocimiento y recuerdo del topónimo forma parte del proceso de identificación con un referente urbano, como penquista. El carácter invisible del referente aporta, además, un carácter casi iniciático a la identidad, porque solo se conoce el nombre (lugar) por transmisión de otro. No deja de ser paradójico que un nombre localizacional (georreferencial) no se vincule con referentes espaciales físicos, sino con nombres dados, que reflejan la vinculación con un legado histórico colectivo. El ejemplo penquista refleja el caso del uso de un locus de control interno (compartido) como locus externo (georreferencial), retomando la teoría de locus de control en psicología (Rotter, 1954).

\section{FIGURA 7 | Parada del tonto: punto de encuentro penquista y en especial de los} estudiantes ("pingüinos"²)

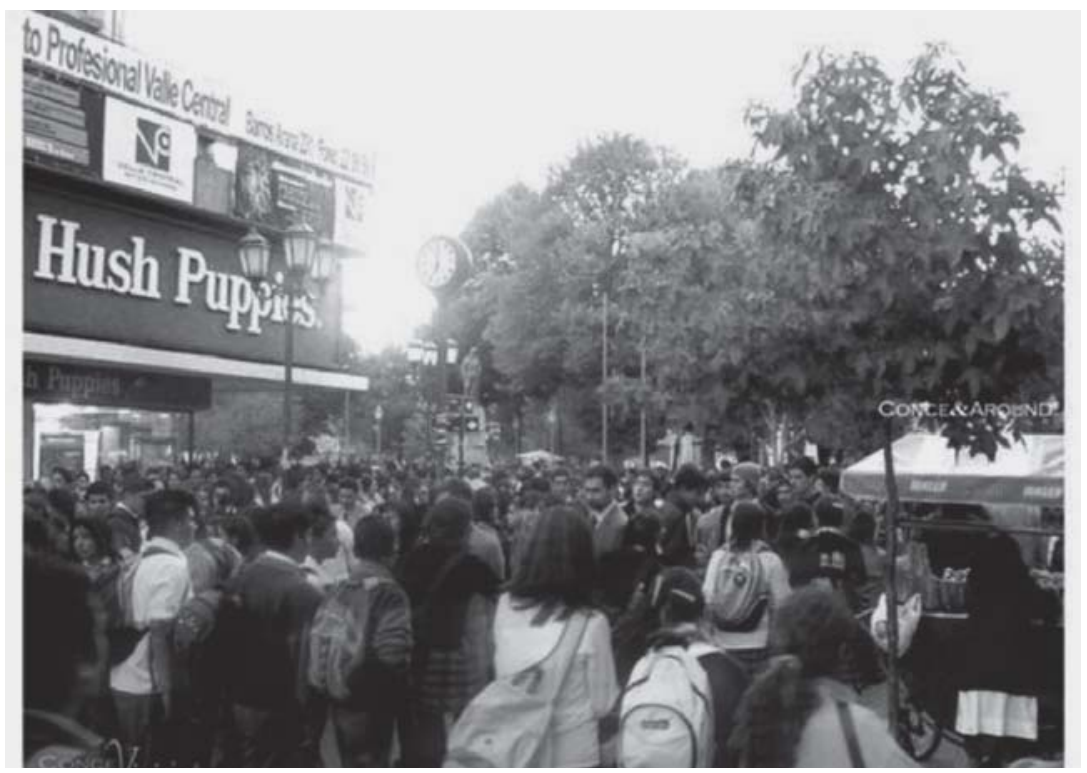

FUENTE HTTP://MIXCOOL.BLOGSPOT.COM/2005/08/CONCEPCIN-LUGAR-PARA-VIVIR.HTML

1 Los comentarios se citan sin ninguna corrección sintáctica y ortográfica.

2 "Pingüinos": apelativo aplicado en Chile a los escolares secundarios que participaron en masivas movilizaciones y paros estudiantiles en el 2006, debido a su uniforme tradicional (camisa blanca, chaqueta oscura). 
El punto de encuentro en Concepción, a diferencia de otros casos, donde tales lugares se vinculan con elementos físicos referenciales - por ejemplo, los ya mencionados café Zurich o el reloj de la plaza del Sol-, debe entenderse como una forma muy particular de adecuación a un lugar. No puede olvidarse que en Concepción el espacio es efímero, a merced de fenómenos naturales de carácter destructivo. Las transformaciones continuas de la morfología urbana, con permanentes reconstrucciones, impiden establecer referentes comunes en el espacio. Esta curiosa situación no ha impedido generar una red de lugares que son transmitidos y que configuran una identidad referencial colectiva.

Esta aproximación al lugar desde el topónimo nos acerca al concepto desarrollado en psicología en el siglo XIX como método loci (localización) o denominado como palacio de los recuerdos (Spence, 1984). Se trata de un método mnemotécnico que se utiliza para entrenar la memoria y que ya está considerado en la retórica clásica (Rhetorica ad Herennium de Cicerón) (Rossi, 2000)3. La transmisión de los referentes, en el caso de Concepción, pasa por un sistema de memorización colectiva transmitida que asocia topónimos y lugares, trazando verdaderos caminos mentales que se reflejan en localizaciones (Carruthers, 1998). El nombre, en este caso, siguiendo la lógica nominalista, ha terminado por crear un referente colectivo, reconocido y que se convierte en identificador real (Rotter, 1954).

Lo interesante del presente caso radica no solo en el proceso de identificación colectiva en torno a ciertos nombres asociados a lugares, con independencia de las formas físicas, sino también en la forma que reviste el patrimonio, como transmisión de un legado vivo que es común a la ciudadanía. La construcción colectiva (Milgram, 1984) se renueva generación tras generación, por parte de una colectividad urbana que se reafirma con la transmisión del legado (memoria de los lugares), se reencuentra como sociedad urbana colectiva tras cada evento telúrico, y a su vez garantiza la supervivencia de los lugares como memoria. El proceso de construcción colectiva significa una reconstrucción definidora de cada generación, que opera compartiendo el referente territorial y redefiniéndolo en el gentilicio. De esta forma, por ejemplo, vemos cómo la antigua Esquina de la puñalá, construida desde una visión del espacio de principios del siglo xx, dominada por el hombre (el varón), se reconvierte en La parada del tonto, como punto de encuentro y referente del mundo escolar, y ello por varias generaciones. Las significaciones han variado, cambiando incluso el nombre del lugar, pero siguen denotando el mismo espacio físico, como herencia de un lugar legado que participa de la permanencia del referente colectivo. La nuevas generaciones reconceptualizan sus lugares de encuentro, a partir de su difusión vía Internet y por medio de plataformas virtuales como Facebook. En este sentido, la inclusión de La parada del tonto como uno de los hitos turísticos de la ciudad por parte de ciertas plataformas virtuales, demuestra el grado de importancia acordado al símbolo por parte de la comunidad local, y a

3 "El método loci consiste en crear un itinerario compuesto de hasta cien lugares en un entorno familiar. Imaginativamente se forman secuencias de objetos, sitios y particularmente estancias de un "palacio mental". Estos objetos se irán asociando con aquello que se desea recordar: la lista de la compra, las gestiones por hacer, las llamadas de teléfono, etcétera” (http://es.wikipedia.org/ wiki/M\%C3\%A9todo_de_loci). 
la vez acentúa el carácter patrimonial del referente territorial, no tanto en este caso desde su dimensión material, sino más bien entendido como legado y símbolo. El compartir ese referente por los medios sociales no hace más que validar su importancia y, en cierto modo, lo valida de forma más institucionalizada y universal.

\section{Al encuentro del lugar}

El aglutinamiento colectivo crea puntos de encuentro que terminan forjando lugares que son simbolizados y convertidos en referente y garantía de la identidad del grupo humano urbano que les ha dado origen. La interacción entre el punto de encuentro y el lugar es casi la concreción misma de la esencia de la urbanidad. El caso del Carfax en Oxford es un excelente ejemplo de cómo una encrucijada de la ciudad medieval (siglo xirI) terminó convirtiéndose en el punto de encuentro y símbolo referencial de sus habitantes (Figuras 8 y 9). La misma etimología de la palabra Carfax alude a la palabra francesa "Carrefour"y al origen latín de "Quadrifurcus" y, con ella, a la idea de partición y cruce. La esquina céntrica, nodo que sirve de referente espacial de localización, se ve rematada por una iglesia, que con su campana y su ulterior reloj aporta la dimensión simbólica del lugar, convirtiéndolo en el punto de encuentro de sus habitantes hasta la actualidad.

La importancia de la permanencia del referente ha sido tan notable que en el caso de Oxford se convirtió en un hito que será considerado y tratado como patrimonio, en -por ejemplo- el proceso de ensanchamiento de la calle que llevó a la reconstrucción, en el siglo XIx, de la misma iglesia y cruce. El punto de encuentro representa un hito en la ciudad de carácter universal, al dar cuenta de la interacción entre la ciudad y su ciudadanía (Figura 8 y 9). Se manifiesta más allá del lugar espacio-temporal, como un hito referencial para la identidad colectiva que debe ser transmitido. Representa, en cierta forma, la esencia de la identidad ciudadana, asociada al lugar y entendida como topoi. Es el argumento vehicular de la identidad de un grupo humano asociado a un lugar, en la interrelación nominal que se ejerce entre la comunidad y su gentilicio. El punto de encuentro es el definidor visible y universal -ya que mantiene una continuidad en el tiempo- de esa estrecha relación que cambia pero permanece intacta como referente específico.

El presente caso de estudio de La parada del tonto, en Concepción, permite evidenciar cómo el lugar termina convirtiéndose en hito ciudadano y adquiere una dimensión patrimonial, al ser considerado como referente de una identidad local urbana. En el caso particular penquista, se da además la curiosa situación de que el hito se vincula a una localización que no se relaciona con elementos espaciales, sino con un nombre dado transmitido por la comunidad. La dimensión del hito se centra no tanto en el valor material o del bien patrimonial, sino en la importancia de la transmisión intergeneracional del legado. El carácter efímero de las formas materiales en un contexto sísmico habitual ha incidido seguramente en esta curiosa adaptación, consiguiendo que el símbolo permanezca en el tiempo, más allá de su forma, gracias a la supervivencia de sus habitantes. 
FIGURAS 8 y 9 | El hito es conservado más allá de su forma

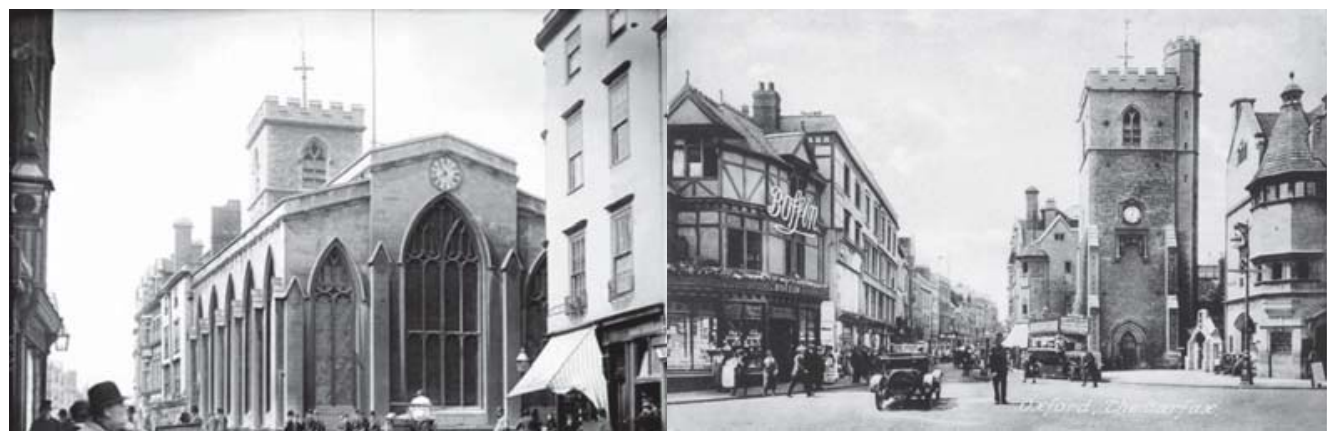

FUENTES HTTP://VIEWFINDER.ENGLISH-HERITAGE.ORG.UK/SEARCH/DETAIL.ASPX?UID=47756 Y HENRY W. TAUNT COLleCtion. EN LA FOTO DE LA IZQUiERDA APARECE EL CARFAX (PUNTO DE ENCUENTRO DE OXFORD, REINO UNIDO), ANTES DE SU TRANSFORMACIÓN POR LA AMPLIACIÓN DE LA CALLE EN I 896. EN LA FOTO DE LA DERECHA, DE LOS AÑOS VEINTE, PERMANECE EL HiTO COLECTIVO EN TODOS SUS ASPECTOS, RESPETADO POR SUS AUTORIDADES

\section{El lugar legado}

En esta situación, es la comunidad (gentilicio) la que garantiza la pervivencia de un referente (topónimo) asociado a un lugar, con independencia de su forma transitoria. La huella de la marca no trasciende en el grupo con el paso del tiempo, como podría ocurrir con un monumento (ejemplo: pirámide en Egipto). En este caso, la salvaguardia del gentilicio por sus habitantes se convierte en hito colectivo; y ellos, en sus guardianes. De todas maneras se mantiene la estrecha relación entre el hito y sus habitantes, variando solo el sentido de la interacción, cuyo objetivo es la permanencia o universalidad del referente.

\section{Un hito universal: topoi}

La indagación en torno a La parada del tonto nos lleva a una reflexión más teórica sobre la importancia de los lugares de encuentro, sus procesos de construcción como hitos colectivos, así como su trascendencia espacio-temporal para convertirse en referentes particulares (locales), pero a la vez universales (referente atemporal). El reloj mundial o Weltzeituhr de la Alexander Platz en Berlín, diseñado en 1969 por Eric John, sintetiza esta doble relación: por un lado, un hito particular; y por otro, la dimensión universal del tiempo astronómico (Figura 10). La dialéctica de lo universal y lo particular queda fraguada en ese reloj que se ha convertido, además, en uno de los puntos de encuentro más concurridos de Berlín. En este caso, vemos una vez más cómo los puntos de encuentro, con independencia de sus particularidades, tienen ciertos elementos comunes, como son su vinculación con nodos, su relación con elementos temporales, así como su carácter de esquina respecto de un punto central.

El retomar el concepto de topoi en la teoría lingüística desarrollada respecto de la retórica ( $\tau$ ó $\pi$ os, en griego lugar, de tópos koinós, lugar común y en plural topoi), nos permite entender, respecto de la geografía, cómo un lugar común puede convertirse en un hito (categoría) y terminar definiendo un referente particular (Ernst, 1953). El hito se convierte igualmente en un espacio topológico desde la definición matemática del topoi (Deligne, 1998) y puede ser entendido como un 
funtor (función de una categoría a otra), al dar continuidad en el tiempo a una identidad (Carnap, 1950). El lugar se convierte en un símbolo que permite hacer congeniar la universalidad de la identidad del referente, respecto de la particularidad excepcional del referente. Esto se sintetiza en la idea de saber encontrar el sentido del lugar y, como decía Jacobs (1958), reconocer en cierto modo al alma del lugar, desde una perspectiva ontológica.

\section{FIgURA 10 El reloj universal de la Alexander Platz}

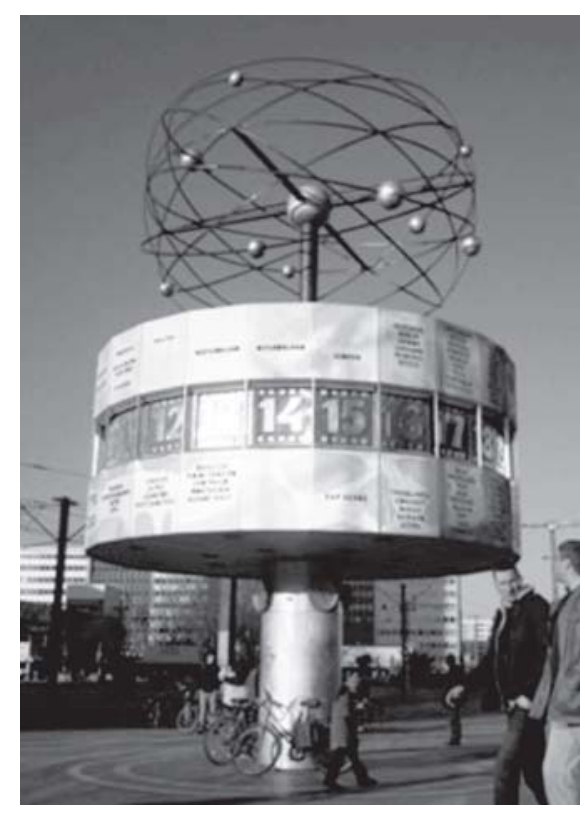

FUENTE HTTP://WWW.ALEMANOL.DE/TURISMO-EN-BERLIN/DIE-WELTZEITUHR/ITEM/EL-RELOJ-MUNDIAL. ESTE LUGAR ES UNO DE LOS PUNTOS DE ENCUENTRO DE BERLÍN (ALEMANia). EL hito SE CONVIERTE EN UN TOPOI DESDE SU DIMENSIÓN UNIVERSAL Y, A LA VEZ, EXCEPCIONAL

\section{Referencias bibliográficas}

Aguilar, M. A. (1990). La construcción de una psicología urbana. Polis - Anuario de Sociología (Universidad Autónoma Metropolitana, Iztapalapa, México), 90, 397-417. En http:// www.journals4free.com/link.jsp?l=35967977

Alarcón, M. (1998). Plaza de la Independencia. Concepción: Editorial Municipalidad de Concepción.

Aragonés, J. I., Corraliza, J. A., Cortés, D. \& Amérigo, M. (1992). Perception of territory and social identity. En Socio-environmental metamorphoses: Builtscape, landscape, ethnoscape, eoroscape (Proceedings 12th Internacional Conference of the IAPs [International Association for People-environments Studies), Chalkidikik, Greece, 11-14 July 1992. Vol. II, 252-259. 
Bettendorff, M. E. (2005). La identidad como memoria y proyecto: Un abordaje transdisciplinar a las construcciones identitarias. Creación y Producción en Diseño y Comunicación (Buenos Aires: Universidad de Palermo), 3, 9-18. En http://fido.palermo.edu/ servicios_dyc/publicacionesdc/archivos/18_libro.pdf

Campos, F. (1985). Concepción en la primera mitad del siglo XX. Santiago: Museo Histórico Nacional.

Carnap, R. (1950). Empiricism, Semantics, and Ontology. Revue Internationale de Philosophie, 4(2), 20-40. En http://www.ditext.com/carnap/carnap.html

Carruthers, M. (1998). The craft of thought. Cambridge, MA: Cambridge University Press.

Corraliza, J. A. (1987). La experiencia del ambiente. Percepción y significado del medio construido. Madrid: Tecnos.

Deligne, P. (1998). Quelques idées maîtresses de l'œuvre de A. Grothendieck. Actes du colloque à la mémoire de Jean Dieudonné (pp. 11-19). Nice : Société Mathématique de France. En http://www.math.jussieu.fr/-leila/grothendieckcircle/deligne.pdf

Ernst, R. C. (1953). European Literature and the Latin Middle Ages. Nueva York, NY: Pantheon Books, 80 .

Francis, R. (1983). Symbols, images and social organization in Urban Sociology. En V. Pons \& R. Francis (Eds.), Urban Social Research: Problems and Prospects (pp. 115-145). Londres: Routledge \& Kegan Paul.

Halbwachs, M. (1968). La Mémoire collective. París: Presses Universitaires de France (PUf).

Jacobs, J. (1958). Downtown is for people. Fortune Classics (April). Reproducido en http:// features.blogs.fortune.cnn.com/2011/09/18/downtown-is-for-people-fortuneclassic-1958/

Jacobs, J. (2004). Dark age ahead. Nueva York: Random House.

Lalli, M. (1988). Urban identity. En D. Canter (Ed.), Environmental Social Psychology (nato ASI Series, Behavioural and Social Sciences, 45), 303-311. Dordrech, The Netherlands: Kluwer Academic Publishers. doi 10.1007/978-94-009-2802-2_26

Lara, H. (1998). La ciudad mártir. Concepción: I. Municipalidad de Concepción.

Louvel, R. (2007). Crónicas y semblanzas de Concepción. Concepción: Sucesión René Louvel Bert. En http://www.bibliotecamunicipaldeconcepcion.cl/libros/cronica/indice.htm

Lynch, K. (1960). The image of the city. Cambridge, Ma: MIT Press.

Lynch, K. (1972). What time is this place? Cambridge MA: MIT Press.

Mazzei de Grazia, L. (Comp.), (2004). Augusto Vivaldi Cichero: Escritos para la construcción de una historia regional. Concepción: Ediciones Escaparate.

Milgram, S. (1984). Cities as social representations. En S. Moscovici \& R. Farr (Eds.), Social Representations (pp. 289-309). Cambridge, MA: Cambridge University Press.

Nogué, J. (Ed.) (2007). La construcción social del paisaje. Madrid: Biblioteca Nueva

Nogué, J. \& Romero, J. (Eds.) (2006). Las otras geografias. València: Tirant lo Blanch.

Nora, P. (2009). Pierre Nora en Les lieux de mémoire. Santiago, Chile: LOM Ediciones, Trilce.

Oliver, C. \& Zapata, F. (1950). Libro de oro de la historia de Concepción. Concepción: Editorial Litográfica.

Pacheco, A. (1997). Historia de Concepción, siglo XX. Concepción: Editorial Universidad de Concepción.

Ricoeur, P (1999). La lectura del tiempo pasado: memoria y olvido. Madrid: Universidad Autónoma de Madrid, Arrecife. 
Rolleri, N. (2007). La torre transparente Recuperación de la memoria: la Municipalidad de Concepción en la Plaza de la Independencia. Concepción: Fondo de Iniciativas Culturales Comunales de la Municipalidad de Concepción.

Rossi, P. (2000). Logic and the art of memory. Chicago, Il: University of Chicago Press.

Rotter, J. B. (1954). Social learning and Clinical Psychology. Nueva York, NY: Prentice-Hall.

Spence, J. D. (1984). The Memory Palace of Matteo Ricci. Nueva York, NY: Viking Penguin.

Valera, S. (1996). Análisis de los aspectos simbólicos del espacio urbano. Perspectivas desde la Psicología Ambiental. Revista de Psicología Universitas Tarraconensis, 18(1), 63-84. En http://www.ub.edu/dppss/valera/1996_Tarraconensis.pdf

Yates, F. A. (1966). The art of memory. Chicago, Il: University of Chicago Press. 